\title{
Illusions of time and extent when the Müller-Lyer figure moves in an aperture
}

\author{
R. H. DAY and F. M. DUFFY \\ Monash University, Clayton, Victoria, Australia
}

\begin{abstract}
Following informal observations, three experiments confirmed that the Müller-Lyer illusion occurs when the composite figure consisting of angles-outward and angles-inward components is progressively exposed in a narrow slit, as in the Zöllner-Parks demonstrations. The illusion of extent was consistent over subjects and about half that with the figure stationary and moving in full view. The experiments showed also that there is a parallel illusion of time, with one of the two equal extents taking apparently longer than the other to pass the slit. The illusion of extent was enhanced or canceled by manipulating the relative velocities of the two components and, therefore, the time taken by them to pass the slit. It is argued that time of exposure in the slit serves as the reference for extent. The illusions of time and extent are attributed to a perceptual compromise between the time taken by an extent to pass the aperture and that of the component itself to do so. The role of time in perceived extent and its possible involvement in visual scanning are considered.
\end{abstract}

Zöllner (1862) was the first to show that when a figure is progressively exposed by passing it behind a narrow aperture so that at any instant only a small part of it is visible the figure is readily recognized and seen as complete. Zöllner noted that the figure appears shortened in the direction of movement at relatively high velocities and lengthened at relatively low ones. He called the phenomenon "anorthoscopic perception." The effect was subsequently confirmed in numerous experiments (see Anstis \& Atkinson, 1967) and later rediscovered by Parks (1965). It is now frequently referred to as the Parks effect. Here it is called the Zöllner-Parks effect.

Perception of a complete figure when it is progressively exposed in a narrow aperture implies that form perception occurs in the absence of a complete retinal image. Parks (1965) argued that the sequential views of the figure were somehow stored, encoded in memory, and reassembled in their original order. An alternative explanation, couched in terms of the extraction of motion and figural features by the visual system, has been proposed more recently by Casco and Morgan (1984). In considering the Parks-Zöllner effect, it is worth bearing in mind that the region of clear vision subtends a visual angle of about $1^{\circ}-2^{\circ}$ of visual angle, thus requiring visual scanning of objects of greater subtense. Therefore, the perceived completeness of a progressively exposed figure could be due

These experiments were undertaken while the first author was in receipt of a grant from the Australian Research Grants Scheme and the second author of a vacation scholarship from Monash University. The paper has benefited considerably from the comments of G. W. Stuart and two anonymous reviewers. The technical assistance of Richard Hobbs, Vladimir Kohout, and Rosemary Williams is gratefully acknowledged. Requests for reprints should be sent to R. H. Day, Department of Psychology, Monash University, Clayton, Victoria 3168, Australia. to the same processes invoked by normal visual scanning. In other words, progressive exposure in a narrow aperture might simply simulate scanning. This view was first put forward by Cattell (1900).

During some informal observations, we noticed that the composite Müller-Lyer (ML) figure consisting of a long and short component also appeared complete when moved behind and at right angles to a narrow vertical slit (Figure 1). In addition, the illusory difference between the two equal extents was clearly evident. The apparent time taken for each extent to pass the aperture seemed also to be different. It can be noted that Hochberg (1968) pointed out that Müller-Lyer figures are clearly recognizable when viewed moving behind a slit. However, he did not comment on the occurrence of the illusion under these conditions.

The ML illusion has not yet been satisfactorily explained (see Coren \& Girgus, 1978; Robinson, 1972). The occurrence of the illusion with continuous, progressive presentation of the figure in a narrow slit and a parallel effect, hitherto unreported, in the time domain are potentially relevant to an explanation. Therefore, we carried out three experiments to check and extend the informal observations. The first was concerned with a comparison between the ML illusion when the figure was progressively exposed by moving it past a slit and when it was stationary and fully visible; the second, with the apparent times taken by the two components to pass the slit; and the third, with the effect on the illusion of speeding up or slowing down each component of the figure.

Although the emphasis in these experiments was on the implications of these new effects for an explanation of the ML illusion, the data are clearly relevant to a closer understanding of the role of time as a basis for perceived extent. This issue is also discussed below. 

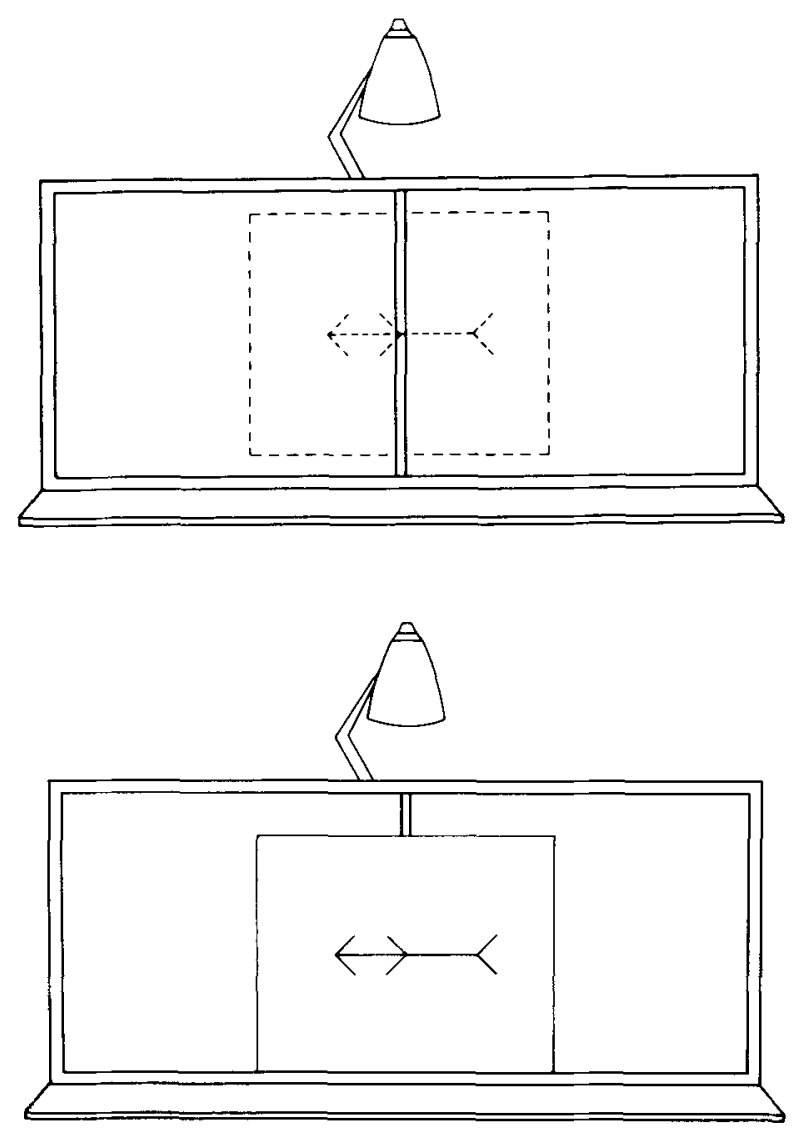

Figure 1. Arrangement for progressively exposing the Müller-Lyer and control figures in a narrow slit (upper) and while stationary and in full view (lower).

\section{METHODS}

\section{Subjects}

The points of subjective equality (PSEs) for the two horizontal extents in the $\mathrm{ML}$ and control (C) figures were established by means of a staircase procedure. This method is precise and reliable but time consuming. Therefore, 6 subjects were considered sufficient for each experiment. They were recruited from among undergraduates, graduate students, and research assistants.

\section{Apparatus}

ML and C figures (Figure 2) were drawn about the centers of $41.5 \times 33.5 \mathrm{~cm}$ rectangular white cards, which were presented either moving horizontally behind a narrow vertical slit or stationary or moving in full view. For the moving (M) condition, the cards were mounted in a frame that moved left or right in guides at selected velocities. The frame was driven by a motor operating through a rack-and-pinion system. The rectangular screen was $99 \mathrm{~cm}$ wide and $42.5 \mathrm{~cm}$ high and had a central slit, $1 \mathrm{~cm}$ wide, extending from top to bottom (Figure 1). The frame could move at the same velocity throughout, or it could be speeded up or slowed down by a mechanical triggering device when the center of the card was exactly centered in the aperture. Screen and stimulus cards were illuminated by an overhead fluorescent source and a specifically directed incandescent lamp so that the card immediately behind the slit was evenly illuminated. The luminance of the matte, gray screen was $196 \mathrm{~cd} / \mathrm{m}^{2}$; that of the card was $358 \mathrm{~cd} / \mathrm{m}^{2}$. For the stationary condition, the cards were placed upright and in full view immediately in front of the central slit (Figure 1). The display was mounted frontoparallel on a table at which the subject sat with his/her chin on a rest. The subject's eyes were at about the same level as the center of the slit. The viewing distance to the screen was $114 \mathrm{~cm}$, at which $1 \mathrm{~cm}$ at the screen subtended a visual angle of about $.5^{\circ}$.

\section{Stimulus Figures}

The ML figures and $\mathrm{C}$ figure in which the two extents are equal are shown in Figures 2. One ML figure (Figure 2A) was used in the first experiment; the other, the Brentano version (Figure $2 \mathrm{C}$ ), was used in a subsidiary experiment. ${ }^{2}$ The figures were drawn with .7-mm black lines on the rectangular white cards so that the central apex or vertical line was always at the center of the card.

The total horizontal extent of the ML figure was $230 \mathrm{~mm}$; that of the $\mathrm{C}$ figure was $200 \mathrm{~mm}$. The oblique lines of the ML figures were $42 \mathrm{~mm}$ long and formed three right angles. The vertical lines in the $\mathrm{C}$ figure were $60 \mathrm{~mm}$, so that the distance from their tips to the horizontal axis was $30 \mathrm{~mm}$. The vertical distance between the tips of the oblique and the horizontal axis was also $30 \mathrm{~mm}$.

The staircase procedure (Cornsweet, 1962; Wetherill, 1963) was used to establish the PSE of the horizontal extents in Figures 2A and 2B. A series of 14 figures was prepared for each. The position of the central angle in the ML figure (Figure 2A) and the central vertical in the $\mathrm{C}$ figure (Figure $2 \mathrm{C}$ ) varied in equal steps from the point of physical equality (PPE). For the ML figures, the step size was $6 \mathrm{~mm}$, and there were 10 steps in the direction to compensate for the illusion and 4 steps in the other direction. For the $\mathrm{C}$ figure, the step size was $1.5 \mathrm{~mm}$, and there were 7 steps in each direction.

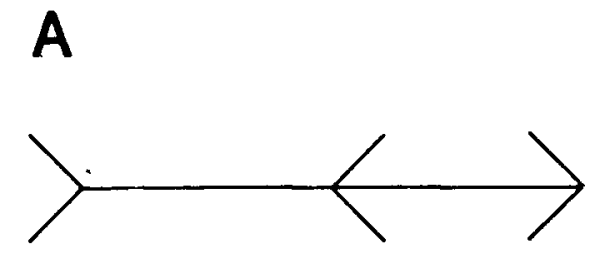

B

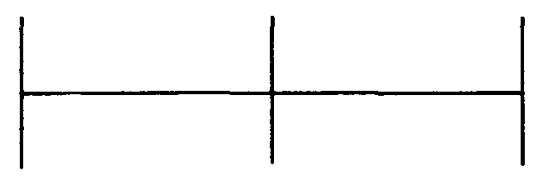

Q
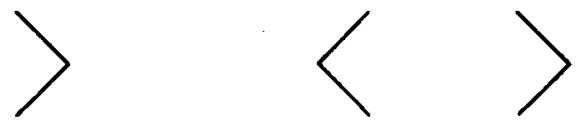

Figure 2. Figures used in the experiments: (A) conventional form of the Mïller-Lyer figure, (B) control figure, and (C) Brentano form of the Müller-Lyer figure. 
Thus, there were 14 versions of each figure. The step sizes and the number of steps were selected following preliminary trials with the two figures. The central apex of the ML figures and the central vertical of the $\mathrm{C}$ figures always coincided with the center of the card since, in Experiment 3, an increase or decrease in velocity was always initiated at that point.

\section{Procedure}

The subject's task when the stimulus figure was presented moving in the slit (Condition $M$ ) was to indicate whether the secondappearing of the two extents between the apexes of the angles (interapical extents) or between the vertical lines (intervertical extents) was longer or shorter in length (Experiments 1 and 3) or in time taken to pass the slit (Experiment 2) than the first. The task, when the figure was presented stationary and in full view (Condition $S$ ), was to estimate extent on the right relative to that on the left. If the estimate of each condition of extent or time was longer, it was shortened by one step for the next presentation; if it was shorter, it was lengthened.

A trial to establish the PSE consisted of two independent staircase series, the first beginning with one extreme position of the central angle or vertical of the figure and the second beginning with the other. The two series were randomly "interleaved" so that the direction of the next step could not be anticipated from the preceding estimate. Presentations of the stimulus figure continued until 10 reversals of judgment (longer to shorter or vice versa) had occurred. The PSE was the mean position of the central angle or vertical at which the reversals occurred relative to the PPE. For the ML figures, the PSE was treated as positive if it was in the direction to compensate for the illusion and as negative if it was in the opposite direction. No illusion is present in the $\mathrm{C}$ figure, and therefore the PSE was arbitrarily treated as positive if it was to the right of the PPE and negative if it was to the left of it. As will be noted, the PSEs for this figure were small enough to be of no account.

\section{EXPERIMENT 1}

The purpose of the first experiment was to establish whether the ML illusion occurs when the figure is progressively presented by moving it past a narrow slit, and, if so, to compare its size with that for the stationary, fully visible figure.

\section{Experimental Conditions and Design}

There were four experimental conditions: the ML figure moving in the slit (ML-M) at $110 \mathrm{~mm} / \mathrm{sec}$ and stationary in full view (ML-S), and the C figure moving at the same velocity (C-M) and stationary (C-S). For ML-M, there were separate trials for the two directions of movement of the figure and the two orders of appearance of the long and short components; for $\mathrm{C}-\mathrm{M}$, there were separate trials for the two directions of movement; for ML-S, there were separate trials for the two positions (right and left) of the long and short components; and for C-S, there was a single trial. Thus, there were nine trials. These were undertaken in a different random order by the 6 subjects.

\section{Results and Discussion}

The PSEs for all 6 subjects for the ML figure were positive under both the $M$ and $S$ conditions. They ranged between 4.05 and $10.65 \mathrm{~mm}$ in Condition ML-M and between 6.90 and $20.10 \mathrm{~mm}$ in Condition ML-S. For the $\mathrm{C}$ figure, two PSEs were positive and four negative in
Condition C-M and three were positive and three negative in Condition C-S. The individual PSEs ranged from -2.40 to $2.03 \mathrm{~mm}$ in Condition $\mathrm{C}-\mathrm{M}$ and from -.90 to $2.85 \mathrm{~mm}$ in Condition C-S. The mean PSEs and their standard deviations are shown in Table 1 . It can be seen that the means for the ML figure were relatively large, with that for Condition ML-S about twice the size of that for Condition ML-M, whereas those for Conditions C-M and $\mathrm{C}-\mathrm{S}$ were negligible. Independent $t$ tests showed that the two means for the ML figure were significantly different from zero $\left[t_{\text {ML-M }}(5)=9.09, p<.005 ; t_{\text {ML-S }}(5)=\right.$ $7.55, p<.005]$, whereas those for the $\mathrm{C}$ figure were not, $\left[t_{\mathrm{C}-\mathrm{M}}(5)=0.06, p>.52 ; t_{\mathrm{C}-\mathrm{S}}(5)=1.50, p>.10\right] . \mathrm{A}$ $2 \times 2$ ANOVA, in which the main factors were stimulus figure (ML and $C$ ) and exposure conditions ( $M$ and $S$ ), showed that both factors were significant $\left[F_{\text {fig }}(1,10)=\right.$ $\left.63.94, p<.001 ; F_{\text {cond }}(1,10)=14.75, p<.003\right]$. The interaction between these two factors also proved to be significant $[F(1,10)=8.43, p<.01]$.

These outcomes confirm that the ML illusion of extent occurs when the figure is moved past a narrow slit so that at any instant only a short section of the figure is visible. ${ }^{1}$ Although the effect occurred with all 6 subjects, it was about half the size of that with the figure stationary and fully visible. That the occurrence of the illusion in Condition ML-M is not due simply to the manner of presentation is evidenced by the absence of an illusory effect for Condition C-M. ${ }^{2}$

\section{EXPERIMENT 2}

The initial observations suggested that the apparent times taken by the equal interapical extents of the ML figure to pass the slit were different. The time taken by the extent with outward-directed angles appeared greater than that with inward-directed angles. This observation has two implications for an explanation of the ML illusion. First, it suggests that the ML effect is a general effect that occurs also in the time domain, and, second, that when the figure moves past an aperture, the time it takes

Table 1

Mean PSEs and Their Standard Deviations for Interapical Extents (Experiment 1) and Their Apparent Duration in the Slit (Experiment 2) When the Müller-Lyer (ML) and Control (C) Figure Were Moving Past the Slit (M) and Stationary in Full View (S)

\begin{tabular}{|c|c|c|c|c|}
\hline & \multicolumn{4}{|c|}{ Conditions } \\
\hline & $\mathbf{M}-\mathbf{M}$ & $\mathbf{C}-\mathbf{M}$ & ML-S & $\mathrm{C}-\mathrm{S}$ \\
\hline \multicolumn{5}{|c|}{ Experiment 1} \\
\hline $\begin{array}{l}\text { Mean PSE (mm) } \\
\text { SD }\end{array}$ & $\begin{array}{l}8.55 \\
2.30\end{array}$ & $\begin{array}{r}.04 \\
1.67\end{array}$ & $\begin{array}{r}14.23 \\
4.62\end{array}$ & $\begin{array}{r}.60 \\
1.40\end{array}$ \\
\hline \multicolumn{5}{|c|}{ Experiment 2} \\
\hline $\begin{array}{l}\text { Mean PSE (msec) } \\
\text { SD }\end{array}$ & $\begin{array}{l}90.25 \\
27.27\end{array}$ & $\begin{array}{r}0 \\
15.52\end{array}$ & & \\
\hline
\end{tabular}


to do so might serve as a reference for extent. Therefore, the purpose of the second experiment was to establish whether, under the same conditions as the first, a ML illusion of time does in fact occur. The question of whether time serves as a reference for extent was taken up in the third experiment.

\section{Conditions and Experimental Design}

The $\mathrm{ML}$ and $\mathrm{C}$ figures were the same as those in Experiment 1. There were two conditions, ML-M and C-M. The 6 new subjects were instructed to estimate whether the duration of the secondappearing interapical extent was greater or less than the firstappearing, that is, whether the second took a longer or a shorter time than the first to pass the slit. The requirement to estimate duration of exposure was strongly emphasized. There were again four trials for Condition ML-M and two for Condition C-M. Each subject underwent the six double-staircase trials in a different random order. Scoring was in milliseconds, with positive and negative values assigned as earlier for estimates of extent.

\section{Results and Discussion}

The PSEs for all 6 subjects in Condition ML-M were positive and greater than those for Condition C-M. Of those for the latter, two were positive and four negative. The mean PSEs and their standard deviations are shown in Table 1. It can be noted that the mean for the ML figure was relatively large and positive and that for the $C$ figure was zero. Statistical treatment of these clear-cut outcomes was not necessary.

The actual times taken by the two physically equal interapical extents of the ML figure to pass the $1-\mathrm{cm}$ slit were the same. However, the apparent time taken by the extent ending in outward-directed angles was greater than that ending in inward-directed angles. The apparent times taken by the two extents in the $\mathrm{C}$ figure were the same. Given that the requirement to estimate time was stressed to the subjects, it can be confidently concluded that when the ML figure is progressively exposed in a narrow aperture an illusion of time paralleling that of extent occurs. The implication of this effect for the spatial illusion was explored further in the last experiment.

\section{EXPERIMENT 3}

Consideration of the stimulus situation when the figure moves past a narrow slit leads to the view that the time taken to pass the slit can serve as a reference for extent. There seems to be no other stimulus information for length. This point may be clarified by considering a more or less commonplace situation. If a long vehicle is viewed passing a narrow window, its apparent length could be estimated only in terms of the time it takes to do so.

If time to pass the slit $i s$ the reference for extent, it follows that the magnitude of the ML illusion of extent would vary as a function of the velocity of the two components. The faster a component moves, the less time it will take to pass and, if the time-reference hypothesis is valid, the shorter it will appear. Likewise, the slower the movement, the greater the apparent extent. Thus, if the long component of the figure were to move slowly and the short com- ponent quickly, the illusion could be expected to increase. On the other hand, if the long component were to move quickly and the short slowly, the illusion could be expected to decrease or even reverse. The purpose of the third experiment was to test these expectations. The two components were presented moving at the same velocity, the long component moving slowly and the short component fast, and the long component moving fast and the short component slowly.

\section{Conditions and Experimental Design}

The slow (S) velocity was $38 \mathrm{~mm} / \mathrm{sec}$, the intermediate velocity (I), $64 \mathrm{~mm} / \mathrm{sec}$, and the fast velocity (F), $90 \mathrm{~mm} / \mathrm{sec}$. In one condition, the long (L) and short (S) components moved at the same velocity (LI-SI), in another, the long component moved slowly and the short component moved fast (LS-SF), and in the third, the long component moved fast and the short component moved slowly (LF-SS). To cancel any effects due to direction of movement or order of appearance of the components, there were four trials under each condition, as for Condition ML-M in Experiment 1.

The order of the 12 trials was differently randomized for each subject. It was expected that if time to pass the slit is the basis of apparent extent, the size of the effect for Condition LI-SI would be about the same as before, for LS-SF greater, and for LF-SS less.

\section{Results and Discussion}

The individual PSEs for Condition LS-SF were consistently greater than those for Condition LI-SI; those for Condition LF-SS were consistently smaller. For Conditions LI-SI and LS-SF, all PSEs were positive; for Condition LF-SS, four were positive and two were negative. The mean PSEs and the standard deviations are shown in Table 2. It can be seen that the mean for Condition LI-SI was about the same as before, that that for LS-SF was about double, and that that for LF-SS was much smaller. Individual $t$ tests showed that although the means for the first two of these conditions were significant, the mean for the last was not $\left[t_{\mathrm{LI}-\mathrm{SI}}(5)=4.50\right.$, $p<.01 ; t_{\mathrm{LS}-\mathrm{SF}}(5)=4.19, p<.01 ; t_{\mathrm{LF}-\mathrm{SS}}(5)=1.16$, $p>$.15]. A one-way ANOVA showed that the effect of the velocities, that is, the effect of duration of time to pass the slit, was significant $[F(2,10)=14.51, p<.01]$. Further analysis, by means of the Newman-Keuls test, showed that the means for LI-SI were different from those for LS-SF and LF-SS and that the latter were different from each other at $p<.01$ or better.

The outcomes of this experiment are unequivocal in showing that the time taken for the components of the composite ML figure to pass the slit determines the size of the illusion. When the time for the long component was relatively long and that of the short component relatively

Table 2

Mean PSEs and Their Standard Deviations for Slow (S), Intermediate (I), and Fast (F) Movement of the Long (L) and Short (S) Components of the ML Figure Moving Past the Slit

\begin{tabular}{lccc} 
& \multicolumn{3}{c}{ Conditions } \\
\cline { 2 - 4 } & LI-SI & LS-SS & LF-SF \\
\hline Mean PSE & 8.93 & 16.43 & 2.93 \\
SD & 4.86 & 9.60 & 6.14 \\
\hline
\end{tabular}


short, the ML illusion of extent was almost twice the size of that when the durations were the same. When the durations for the two components were reversed, the illusion was negligible.

\section{GENERAL DISCUSSION}

The informal observations that led to these experiments demonstrate that, like other figures, the ML figure is perceived as complete when it moves past a narrow slit. The outcomes of the first experiment and a subsidiary experiment ${ }^{2}$ are clear in showing that when either the conventional or Brentano form of the ML figure is presented in this way, the illusion of extent persists. Under the condition of Experiment 1, the illusion was about half that with the moving or stationary figure in full view. A parallel illusion occurs in respect to time taken by the interapical extents to pass the slit. The illusion of extent can be enhanced or canceled by manipulating the velocities of the two components of the figure so that the times taken by each to pass the slit are different.

These outcomes are of interest here mainly from the standpoint of an explanation of the ML illusion. The results of Experiment 2 are of particular interest in showing that in aperture presentation, the illusion occurs in time as well as in space. An adequate explanation can therefore be reasonably expected to account for both the temporal and spatial forms of the illusion. An explanation in terms of perceptual compromise between two or more states of affairs in the stimulus array is proposed. The principle of perceptual compromise has been developed mainly in relation to the Poggendorff misalignment effect (Day \& Jolly, 1987; Day \& Kasperczyk, 1985; Day, Watson, \& Jolly, 1986). The principle is that when the stimulus array incorporates two or more references for an object property, for example, size, straightness, or alignment, the perceived property is a compromise between them. For example, there are two references for alignment in the conventional form of the Poggendorff figure, the axis of the oblique elements and that of the vertical parallels. The oblique elements are aligned in the first but misaligned relative to the second. The elements appear to be slightly misaligned in the direction of their actual misalignment relative to the vertical axis. That is to say, there is a compromise in perception between the state of alignment relative to one axis and of misalignment relative to another.

In the case of the ML figure in aperture presentation, it is convenient first to consider the illusion of time. The references involved are the actual times taken by the interapical extents, the extents that flank them, and, in the case of the component with outward-directed angles, the whole component. For the component with outward-directed angles, the times taken by the flanking extents and the component itself to pass the slit are greater than the time taken by the interapical extent. It is proposed that a compromise occurs in perception between these states of affairs, so that the time for the interapical extent appears greater than it is. For the component with inward-directed angles, the time taken by the flanking extents is less than that taken by the interapical extent, so that the apparent time taken by the latter is less than its actual time. With the latter figure, the times for the interapical extent and the component are the same. In short, for each component there is a shift in perception away from the time taken by the target extent to pass the slit toward that taken by the longer or shorter flanking extent and, for the component with outward-directed angles, also toward that of the whole component.

The results from Experiment 3 show that with aperture presentation perceived extent is determined by the time taken to pass the slit. It follows that if the apparent time taken by the extents to pass are different, their apparent extents will differ accordingly. In short, it is contended here that the perceptual compromise that results in the temporal illusion with the ML figure give rise also to the illusion of spatial extent.

Clearly, this argument has implications for an explanation of the conventional ML illusion with fully visible stationary figures. While it is not appropriate to pursue this matter in detail here, it is suggested that the illusion is the outcome of a compromise in perception between the reference provided by the actual extents, on the one hand, and that provided by the actual longer or shorter flanking and component extents, on the other. That is to say, the same compromises occur between extents as between times in aperture presentation.

Three subsidiary issues warrant brief comment. First, if spatially separated points are presented in alternation, the time interval between them is a significant determinant of the apparent extent between them. This is the tau effect (Collyer, 1977; Helson, 1930; Helson \& King, 1931). As with the Zöllner-Parks arrangement, the actual extent in the tau situation is never fully visible at one time. This raises the question as to whether apparent extent in the Zöllner-Parks and tau effects are essentially the same.

The second issue concerns the integrative process involved in the perception of a complete figure from a sequence of fragments. Shimojo and Richards (1986) have argued that the process requires that the observer know in which direction and at what speed the mainly occluded figure is traveling. They conclude that observers impose the constraint of a rigid body moving at constant speed. In the experiments described here, the ML figure was consistently seen as complete. Yet, between the disappearance of one angle and the appearance of the other the slit contained only a short section of horizontal line, which was apparently stationary, or nothing at all. It is for this reason that we have taken the time to pass the slit rather than the velocity of movement, to be the basis of apparent extent. It is, of course, possible that the observer converts time into constant velocity, but we have no evidence that this is so.

The last issue is a speculation about the role of visual scanning in perceived extent. It is suggested in the introduction that perceived completeness with aperture 
presentation could be due to the same processes invoked by visual scanning with a narrow $\left(1^{\circ}-2^{\circ}\right)$ field of clear vision, a proposal first made by Cattell (1900). If this interpretation is valid, it is conceivable that the time rather than the extent of visual scanning of objects is a basis for perceived extent.

\section{REFERENCES}

ANSTIS, S. M., ATKINson, J. (1967). Distortions in moving figures viewed through a stationary slit. American Journal of Psychology, $80,572-585$

Casco, C., Morgan, M. (1984). The relationship between space and time in the perception of a stimulus moving behind a slit. Perception, 13, 429-441.

Cattell, J. M. (1900). On relations of time and space in vision. Psychological Review, 7, 325-343.

Collyer, C. E. (1977). Discrimination of spatial and temporal intervals defined by three light flashes: Effects of spacing on temporal judgments and of timing on spatial judgments. Perception \& Psychophysics, 21, 357-364.

COREN, S., \& Girgus, J. S. (1978). Seeing is deceiving: The psychology of visual illusions. Hillsdale, NJ: Erlbaum.

CornsweEt, J. N. (1962). The staircase method in psychophysics. American Journal of Psychology, 75, 485-491.

DAY, R. H., \& JoLLY, W. J. (1987). A note on apparent displacement of lines and dots on oblique parallels. Perception \& Psychophysics, 41, 187-189.

DAY, R. H., \& KASPERCZYK, R. T. (1985). Apparent displacement of lines and dots in a parallel-line figure: $A$ clue to the basis of the Poggendorff effect. Perception \& Psychophysics, 38, 74-80.

Day, R. H., Watson, W., Jolly, W. J. (1986). The Poggendorff displacement effect with only three dots. Perceptions \& Psychophysics, 39, 351-354.

HeLson, H. (1930). The tau effect-an example of psychological relativity. Science, 71, 536-537.

Helson, H., \& KING, S. N. (1931). The tau effect: An example of psychological relativity. Journal of Experimental Psychology, 14, $202-217$.
Hochberg, J. (1968). In the mind's eye. In R. N. Haber (Ed.), Contemporary theory and research in visual perception. New York: Holt, Rinehart \& Winston.

PARKs, T. E. (1965). Post-retinal visual storage. American Journal of Psychology, 78, 145-147.

RoBinson, J. O. (1972). The psychology of visual illusion. London: Hutchinson.

Shimojo, S., \& RichaRDS, W. (1986). "Seeing" shapes that are almost totally occluded: A new look at Parks's camel. Perception \& Psychophysics, 39, 418-426.

WeTHERILL, G. B. (1963). Sequential estimation of quantal response curves. Journal of the Royal Statistical Society, 25, 1-48.

ZöLLNER, F. (1862). Über eine neue Art anorthoskopischer Zerrbilder. Annalen der Physik, Poggendorf's Annalen, 117, 477-484.

\section{NOTES}

1. Since the comparison was made between the magnitude of the $M-L$ illusion when the figure was viewed moving in a slit and when stationary in full view, the question arose concerning its magnitude when the figure is moving in full view. A subsidiary experiment (with another 6 subjects), in which the figure was moving and stationary while fully visible, showed that there is no difference between the two conditions. The means were $13.35 \mathrm{~mm}(S D=4.78)$ for the stationary figure and $13.50 \mathrm{~mm}(S D=3.96)$ for the moving figure. The former accords closely with the same condition in Experiment 1.

2. It was possible that the illusion for Condition ML-M was due to the figure's having been viewed while fully visible in Condition ML-S. This was checked in a second subsidiary experiment with 6 new subjects. The Brentano form of the figure (Figure 2C) was used in Condition ML-M only. The mean of $10.84 \mathrm{~mm}(S D=8.30)$ was slightly greater than before and significant $[t(5)=3.20, p<.05]$. This outcome rules out the possibility of an effect of Condition ML-S on ML-M, and shows that the illusion persists in the Brentano form of the figure with aperture presentation.

(Manuscript received July 20, 1987 ; revision accepted for publication February 20, 1988.) 